\title{
Correlation functions for one-dimensional interacting fermions with spin-orbit coupling
}

\author{
Aníbal Iucci* \\ Instituto de Física La Plata. Departamento de Física, Facultad de Ciencias Exactas, \\ Universidad Nacional de La Plata. CC 67, 1900 La Plata, Argentina \\ and Consejo Nacional de Investigaciones Cientificas y Técnicas, Argentina
}

(Received 1 November 2002; revised manuscript received 7 April 2003; published 14 August 2003)

\begin{abstract}
We compute correlation functions for one-dimensional electron systems in which spin and charge degrees of freedom are coupled through spin-orbit coupling. Charge density waves, spin density waves, and singlet- and triplet-superconducting fluctuations are studied. We show that the spin-orbit interaction modifies the exponents and the phase diagram of the system, changing the dominant fluctuations and making new susceptibilities diverge for low temperature.
\end{abstract}

DOI: 10.1103/PhysRevB.68.075107

PACS number(s): 71.10.Pm

In the past two decades there have been intense efforts in studying quasi-one-dimensional electron systems (Q1DES's). This interest has its origin in the simplicity of the models which describe them and, at the same time, in the possibility of making contact with experiments. Examples of these Q1DES's of recent construction are carbon nanotubes, ${ }^{1}$ conducting polymers, ${ }^{2}$ and semiconductor heterostructures. ${ }^{3}$ From the theoretical point of view the simplest formulation of a Q1DES is given by the Tomonaga-Luttinger ${ }^{4,5}$ model which describes the major qualitative features of interacting Q1DES's such as the spin-charge separation and the nonuniversal exponents in the decay law of correlation functions.

In realistic situations the electrons are moving in electric fields inside the materials: microscopic and macroscopic ones, the latter responsible for confining the electrons to a reduced region of space. As a consequence it appears a magnetic field in the rest frame of the electron which couples with its intrinsic magnetic moment and breaks spin-rotation $\mathrm{SU}(2)$ symmetry. This is known as a spin-orbit (SO) interaction (or spin-orbit coupling). Despite its relativistic origin, this interaction has an important effect on existing twodimensional electron gases (2DEG's) such as GaAs/AlGaAs (Refs. 6 and 7) and InGaAs/InAlAs (Refs. 8 and 9) heterostructures. It is responsible for the modification of their band structure by lifting the spin degeneracy and for positive magnetoresistance effects, known as weak antilocalization. ${ }^{10}$

In Q1DES's there exists an additional potential, responsible for confining the electrons in a narrow channel patterned in 2DEG heterostructures. ${ }^{11}$ Although as far as we know there is no experimental evidence or measures of the strength of the SO coupling resulting from such a potential, theoretical work indicates that it affects quantitatively the splitting energy behavior as a function of the wave vector $k$ and produces an asymmetric deformation of each spin branch; i.e., the Fermi velocities take different values for different directions of motion. ${ }^{12}$

In Ref. 13 the following Hamiltonian was proposed as a model for a Q1DES with SO coupling:

$$
H=H_{0}+H_{\text {int }},
$$

where the noninteracting Hamiltonian is

$$
\begin{aligned}
H_{0}= & v_{1} \sum_{k}\left[\left(k+k_{1}\right) c_{k R \uparrow}^{\dagger} c_{k R \uparrow}-\left(k-k_{1}\right) c_{k L \downarrow}^{\dagger} c_{k L \downarrow}\right] \\
& +v_{2} \sum_{k}\left[\left(k+k_{2}\right) c_{k R \downarrow}^{\dagger} c_{k R \downarrow}-\left(k-k_{2}\right) c_{k L \uparrow}^{\dagger} c_{k L \uparrow}\right] .
\end{aligned}
$$

It consists of a modified Tomonaga-Luttinger model, which takes into account the asymmetry in the spectrum for each spin branch, making $v_{1} \neq v_{2}$ (and $\left.k_{1} \neq k_{2}\right)$. $c_{k r s}^{\dagger}$ creates a right-going $(r=+1)$ or left-going $(r=-1)$ electron. The interacting Hamiltonian describes forward-scattering electron-electron interactions and has a standard form. ${ }^{5} \mathrm{Um}$ klapp and backscattering terms are irrelevant if we are far from half filling in the former case, and we restrict ourselves to repulsive interactions in the latter.

In this article we compute correlation functions for charge-density-wave (CDW), spin-density-wave (SDW) $4 k_{F}$ charge-density-wave $\left(4 k_{F}\right)$, and singlet- and tripletsuperconductivity (SS and TS) operators for the model presented above. The correlation functions for these operators are well known in the case of zero spin-orbit coupling, ${ }^{5,14,15}$ including logarithmic correction factors ${ }^{16,17}$ and time and temperature dependence. ${ }^{15}$ We extend these calculations to the case in which SO interactions are present and study how the exponents of their algebraic decay are modified. We find interesting modifications of the phase diagram of the system when SO interactions are present. For certain regions of parameter space, SO coupling changes the dominant fluctuations and makes new susceptibilities diverge for low temperature.

The Hamiltonian (1) can be studied by the use of bosonization technique ${ }^{5,18}$ as in Ref. 13. For convenience we shall define an average velocity $v_{0}=\left(v_{1}+v_{2}\right) / 2$ and the difference $\delta v=v_{2}-v_{1}$, and the same for the Fermi momentum $k_{0}=\left(k_{1}+k_{2}\right) / 2$ and $\delta k=k_{2}-k_{1}$. If we introduce the usual phase fields $\phi_{\rho}\left(\phi_{\sigma}\right)$ for charge (spin) degrees of freedom and the dual field $\Pi_{\rho}\left(\Pi_{\sigma}\right)$, the Hamiltonian can be represented as 


$$
\begin{aligned}
H= & \frac{v_{\rho}}{2} \int d x\left[\frac{1}{K_{\rho}}\left(\partial_{x} \phi_{\rho}\right)^{2}+K_{\rho} \Pi_{\rho}^{2}\right] \\
& +\frac{v_{\sigma}}{2} \int d x\left[\frac{1}{K_{\sigma}}\left(\partial_{x} \phi_{\sigma}\right)^{2}+K_{\sigma} \Pi_{\sigma}^{2}\right] \\
& +\delta v \int d x\left[\left(\partial_{x} \phi_{\rho}\right) \Pi_{\sigma}+\left(\partial_{x} \phi_{\sigma}\right) \Pi_{\rho}\right] .
\end{aligned}
$$

$v_{\rho, \sigma}$ are the propagation velocities of the spin and charge collective modes of the decoupled model $(\delta v=0)$, and $K_{\rho, \sigma}$ are the stiffness constants. The spin-orbit interaction appears as an effect that breaks the spin-charge separation, which is revealed as the presence of a third term in the last equation. Nevertheless, the Hamiltonian (3) can still be diagonalized in terms of two new phase fields which contain a mixture of spin and charge degrees of freedom. The propagation velocities of these collective modes are

$$
\begin{aligned}
v_{ \pm}^{2}= & \frac{v_{\sigma}^{2}+v_{\rho}^{2}}{2}+\delta v^{2} \\
& \pm \sqrt{\left(\frac{v_{\rho}^{2}-v_{\sigma}^{2}}{2}\right)^{2}+\delta v^{2}\left[v_{\sigma}^{2}+v_{\rho}^{2}+v_{\rho} v_{\sigma}\left(\frac{K_{\rho}}{K_{\sigma}}+\frac{K_{\sigma}}{K_{\rho}}\right)\right]}
\end{aligned}
$$

As $\delta v \rightarrow 0, v_{+} \rightarrow \max \left(v_{\rho}, v_{\sigma}\right)$ and $v_{-} \rightarrow \min \left(v_{\rho}, v_{\sigma}\right)$. As $\delta v$ increases, $v_{-}$decreases until it vanishes at the points

$$
\begin{aligned}
& \delta v_{\rho}^{2}=v_{\rho} v_{\sigma} \frac{K_{\sigma}}{K_{\rho}}, \\
& \delta v_{\sigma}^{2}=v_{\rho} v_{\sigma} \frac{K_{\rho}}{K_{\sigma}} .
\end{aligned}
$$

At these points, the freezing of the lower bosonic mode is accompanied by a divergence in the charge and spin response functions. The static charge compressibility $\kappa$ diverges at $\delta v=\delta v_{\rho}$ and at $\delta v=\delta v_{\sigma}$ occurs a divergence of the static spin susceptibility $\chi$. They behave as

$$
\begin{gathered}
\kappa=\kappa_{0}\left[1-\frac{\delta v}{\delta v_{\rho}}\right]^{-1}, \quad \kappa_{0}=\frac{2 K_{\rho}}{\pi v_{\rho}}, \\
\chi=\chi_{0}\left[1-\frac{\delta v}{\delta v_{\sigma}}\right]^{-1}, \quad \chi_{0}=\frac{2 K_{\sigma}}{\pi v_{\sigma}},
\end{gathered}
$$

where $\kappa_{0}$ and $\chi_{0}$ are the values of $\kappa$ and $\chi$ in the absence of SO coupling. Beyond these points the susceptibilities become negatives. This behavior of the static response functions together with the vanishing of the collective-mode velocity indicates that the system becomes unstable ${ }^{5,19}$ and undergoes a first-order phase transition. ${ }^{17}$ For $K_{\rho}>K_{\sigma}, \delta v_{\rho}$ turns out to be lower than $\delta v_{\sigma}$, and as $\delta v$ grows from the zero, a physical divergence takes place in the charge compressibility. This instability is known as phase separation and has been shown to occur in the extended Hubbard model ${ }^{20}$ and in the $t-J$ model. ${ }^{21}$ In the case that $K_{\rho}<K_{\sigma}$, the instability takes place in the spin subsystem and is related to the so-called metamagnetic transition, observed, for instance, in the quasi-one-dimensional compound $\mathrm{Ba}_{3} \mathrm{Cu}_{2} \mathrm{O}_{4} \mathrm{Cl} 2 .^{22}$ It also arises in the phase diagram of the $X X Z$ model with next-to-nearest neighbors. ${ }^{23}$ In the presence of a chemical potential (magnetic field), the region where $\kappa(\chi)$ is negative is associated with the coexistence of two phases with different hole concentration (magnetization). The divergence of $\kappa$ was found in other models with asymmetric dispersion. ${ }^{24}$

Let us now focus our attention on the correlation functions. Our interest in this work is to obtain their space-time and temperature $T=1 / \beta$ behavior. The operators for CDW, $\mathrm{SDW}, 4 k_{F}, \mathrm{SS}$, and TS fluctuations in their bosonized form are

$$
\mathcal{O}_{\mathrm{CDW}}=\frac{2}{\pi a} \cos \left(2 k_{0} x+\sqrt{2 \pi} \phi_{\rho}\right) \cos \sqrt{2 \pi} \phi_{\sigma},
$$

$$
\mathcal{O}_{4 k_{F}}=\frac{1}{(\pi a)^{2}} \cos \left(4 k_{0} x+\sqrt{8 \pi} \phi_{\rho}\right),
$$

$$
\mathcal{O}_{\mathrm{SDW}, x}=\frac{2}{\pi a} \cos \left(2 k_{0} x+\sqrt{2 \pi} \phi_{\rho}\right) \cos \left(\delta k x+\sqrt{2 \pi} \theta_{\sigma}\right),
$$

$$
\mathcal{O}_{\mathrm{SDW}, y}=\frac{2}{\pi a} \cos \left(2 k_{0} x+\sqrt{2 \pi} \phi_{\rho}\right) \sin \left(\delta k x+\sqrt{2 \pi} \theta_{\sigma}\right) \text {, }
$$

$$
\mathcal{O}_{\mathrm{SDW}, z}=\frac{2}{\pi a} \sin \left(2 k_{0} x+\sqrt{2 \pi} \phi_{\rho}\right) \sin \sqrt{2 \pi} \phi_{\sigma}
$$

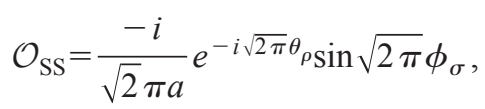

$$
\begin{aligned}
& \mathcal{O}_{\mathrm{TS}, 0}=\frac{1}{\sqrt{2} \pi a} e^{-i \sqrt{2 \pi} \theta_{\rho}} \cos \sqrt{2 \pi} \phi_{\sigma}, \\
& \mathcal{O}_{\mathrm{TS}, \pm 1}=\frac{1}{2 \pi a} e^{ \pm i \delta k x} e^{-i \sqrt{2 \pi}\left(\theta_{\rho} \pm \theta_{\sigma}\right)},
\end{aligned}
$$

where $a$ is a short distance cutoff and $\theta_{\nu}$ is related to the conjugated field $\Pi_{\nu}$ by the relation $\Pi_{\lambda}=\partial_{x} \theta_{\lambda}$.

The correlation functions are defined as

$$
R_{i}(x, \tau ; \beta)=\left\langle\mathcal{T}_{\tau} \mathcal{O}_{i}(x, \tau) \mathcal{O}_{i}^{\dagger}(0,0)\right\rangle,
$$

where $\mathcal{T}_{\tau}$ is the (imaginary) time-ordering operator. These objects were calculated in the path-integral framework within the Matsubara imaginary-time formalism and the results are 


$$
\begin{aligned}
& \left.R_{\mathrm{CDW}}(x, \tau ; \beta)=R_{\mathrm{SDW}, z}(x, \tau ; \beta)=\frac{\cos 2 k_{0} x}{2(\pi a)^{2}}\left(z_{+} \overline{z_{+}}\right)^{-\left(K_{\rho} \nu_{+}^{\rho}+K_{\sigma} \nu_{+}^{\sigma}\right) / 2\left(z_{-} \bar{z}_{-}\right.}\right)^{-\left(K_{\rho} \nu_{-}^{\rho}+K_{\sigma} \nu_{-}^{\sigma}\right) / 2}\left[\left(\frac{\overline{z_{+}} z_{-}}{z_{+} \overline{z_{-}}}\right)^{H \operatorname{sgn}(x \tau)}+\mathrm{H} . \mathrm{c} .\right] \\
& R_{\mathrm{SDW}, x y}(x, \tau ; \beta)=\frac{\cos 2 k_{1} x}{2(\pi a)^{2}}\left(z_{+} \bar{z}_{+}\right)-\left(K_{\rho} \nu_{+}^{\rho}+\mu_{+}^{\sigma} / K_{\sigma}\right) / 2-\theta_{+}^{\sigma}\left(z_{-} \bar{z}_{-}\right)-\left(K_{\rho} \nu_{-}^{\rho}+\mu_{-}^{\sigma} / K_{\sigma}\right) / 2-\theta_{-}^{\sigma} \\
& \left.+\frac{\cos 2 k_{2} x}{2(\pi a)^{2}}\left(z_{+} \bar{z}_{+}\right)^{-\left(K_{\rho} \nu_{+}^{\rho}+\mu_{+}^{\sigma} / K_{\sigma}\right) / 2+\theta_{+}^{\sigma}\left(z_{-} \bar{z}_{-}\right)}\right)^{-\left(K_{\rho} \nu_{-}^{\rho}+\mu_{-}^{\sigma} / K_{\sigma}\right) / 2+\theta_{-}^{\sigma}}, \\
& \left.R_{4 k_{F}}(x, \tau ; \beta)=\frac{\cos 4 k_{0} x}{2(\pi a)^{4}}\left(z_{+} \bar{z}_{+}\right)^{-2 K_{\rho} \nu_{+}^{\rho}\left(z_{-} \bar{z}_{-}\right.}\right)^{-2 K_{\rho} \nu_{-}^{\rho}}
\end{aligned}
$$

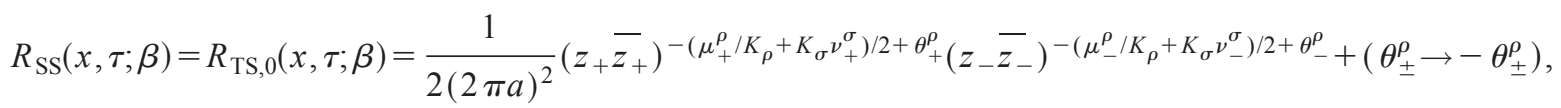

$$
\begin{aligned}
& R_{\mathrm{TS}, \pm 1}(x, \tau ; \beta)=\frac{e^{ \pm i \delta k x}}{(2 \pi a)^{2}}\left(z_{+} \overline{z_{+}}\right)^{-\left(\mu_{+}^{\rho} / K_{\rho}+\mu_{+}^{\sigma} / K_{\sigma}\right) / 2}\left(z_{-} \overline{z_{-}}\right)-\left(\mu_{-}^{\rho} / K_{\rho}+\mu_{-}^{\sigma} / K_{\sigma}\right) / 2\left(\frac{\overline{z_{+}} z_{-}}{\overline{z_{+}}}\right)^{ \pm G \operatorname{sgn}(x \tau)},
\end{aligned}
$$

where

$$
\begin{aligned}
& z_{ \pm}=\frac{\sin \frac{\pi}{v_{ \pm} \beta}\left(v_{ \pm}|\tau|+\epsilon+i x\right)}{\sin \frac{\pi \epsilon}{v_{ \pm} \beta}} \\
& \overline{z_{ \pm}}=\frac{\sin \frac{\pi}{v_{ \pm} \beta}\left(v_{ \pm}|\tau|+\epsilon-i x\right)}{\sin \frac{\pi \epsilon}{v_{ \pm} \beta}}
\end{aligned}
$$

and the exponents depend on the the stiffness constants multiplied by the factors that include mode velocity dependences. They are given by

$$
\begin{gathered}
\nu_{ \pm}^{\lambda}= \pm \frac{v_{\lambda}}{v_{ \pm}} \frac{v_{ \pm}^{2}-v_{-\lambda}^{2}\left(1-\delta v^{2} / \delta v_{-\lambda}^{2}\right)}{v_{+}^{2}-v_{-}^{2}}, \\
\mu_{ \pm}^{\lambda}= \pm \frac{v_{\lambda}}{v_{ \pm}} \frac{v_{ \pm}^{2}-v_{-\lambda}^{2}\left(1-\delta v^{2} / \delta v_{\lambda}^{2}\right)}{v_{+}^{2}-v_{-}^{2}}, \\
\theta_{ \pm}^{\lambda}= \pm \frac{\delta v}{v_{ \pm}} \frac{v_{ \pm}^{2}-\left(\delta v_{\lambda}^{2}-\delta v^{2}\right)}{v_{+}^{2}-v_{-}^{2}}
\end{gathered}
$$

with $\lambda=\rho, \sigma$ and

$$
\begin{gathered}
H=\delta v \frac{K_{\rho} v_{\rho}+K_{\sigma} v_{\sigma}}{v_{+}^{2}-v_{-}^{2}}, \\
G=\delta v \frac{v_{\rho} / K_{\rho}+v_{\sigma} / K_{\sigma}}{v_{+}^{2}-v_{-}^{2}} .
\end{gathered}
$$

Here $\nu_{ \pm}^{\lambda}$ and $\mu_{ \pm}^{\lambda}$ are positive, and $\theta_{ \pm}^{\lambda}, G$, and $H$ have the same sign as $\delta v$.

In the model with zero $\mathrm{SO}$ coupling $\mathrm{SU}(2)$ symmetry can be restored by imposing the constraint $K_{\sigma}=1$, which emerges naturally if the model under study is the continuum limit of a lattice model with only charge density interactions. In this case this is not possible; the $\mathrm{SU}(2)$ symmetry stays broken even for $K_{\sigma}=1$ as revealed by the differences in the decays between SDW operator correlation functions in the $z$ direction and in the $x, y$ directions. As in the zero SO case, correlation functions for SDW operators in the $z$ direction and CDW operators are equal, and the same happens with TS, 0 , and SS operators. This degeneracy is broken by logarithmic corrections that arise if irrelevant backscattering or umklapp terms are included. ${ }^{16}$

An interesting point to observe is the appearance of two terms in the SDW, xy correlation functions [Eq. (19)] where the modulations have different frequencies and decay with different exponents. As $\theta_{ \pm}^{\lambda}$ has the same sign as $\delta v$ [see Eq. (27) and the comment below Eq. (29)] for $v_{2}>v_{1}\left(v_{2}\right.$ $\left.<v_{1}\right)$ the dominant term is the one with frequency $k_{2}\left(k_{1}\right)$. In other words the biggest frequency dominates. Also $R_{\mathrm{TS}, \pm 1}$ becomes oscillating.

Up to here we have obtained very general formulas for space-time and temperature-dependent correlation functions for the model under analysis. We can gain physical insight by observing the algebraic decay of the instantaneous correlation functions at zero temperature and studying how the exponents get modified from the zero SO case. The functions behave as

$$
R_{i}(x) \sim|x|^{-2+\alpha_{i}}
$$

The exponents $\alpha_{i}^{\prime}$ s determine the divergence of the corresponding Fourier space susceptibility as $T \rightarrow 0, \quad \chi_{i}(T)$ 


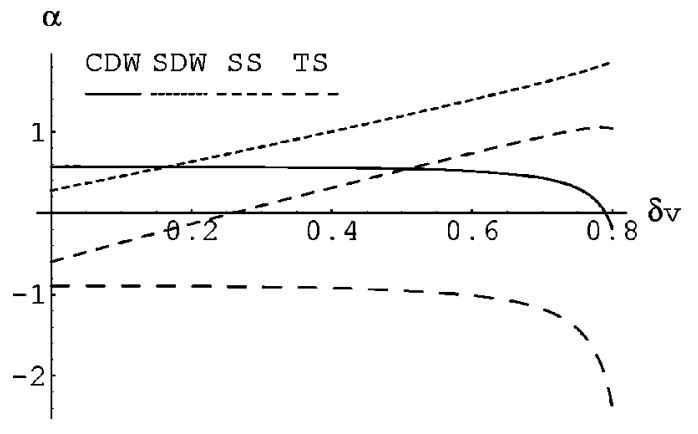

FIG. 1. Behavior of the exponents $\alpha_{i}^{\prime} \mathrm{s}$ as a function of $\delta v$ (in units of $v_{0}$ ). For $v_{\rho}=1.2 v_{0}, v_{\sigma}=0.8 v_{0}, K_{\rho}=0.6$, and $K_{\sigma}=0.85$. For $\delta v \geq 0.16$, SDW, $x y$ fluctuations become dominant, and for $\delta v \gtrsim 0.25, \alpha_{\mathrm{SS}}$ becomes positive and $\chi_{\mathrm{SS}}$ divergent for $T \rightarrow 0$.

$\sim T^{-\alpha_{i} .}$. This makes these instabilities of a completely different nature than the ones described in Eqs. (7) and (8). The expressions obtained for the $\alpha_{i}$ are

$$
\begin{gathered}
\alpha_{\mathrm{CDW}}=\alpha_{\mathrm{SDW}, z}=2-K_{\rho} \nu^{\rho}-K_{\sigma} \nu^{\sigma}, \\
\alpha_{\mathrm{SDW}, x}=\alpha_{\mathrm{SDW}, y}=2\left(1+\left|\theta^{\sigma}\right|\right)-K_{\rho} \nu^{\rho}-\mu^{\sigma} / K_{\sigma}, \\
\alpha_{\mathrm{SS}}=\alpha_{\mathrm{TS}, 0}=2\left(1+\left|\theta^{\rho}\right|\right)-\mu^{\rho} / K_{\rho}-K_{\sigma} \nu^{\sigma}, \\
\alpha_{\mathrm{TS}, \pm 1}=2-\mu^{\rho} / K_{\rho}-\mu^{\sigma} / K_{\sigma} .
\end{gathered}
$$

These are the new exponents, which retain the same structure as in the zero SO coupling, but modified by the factors

$$
\begin{gathered}
\mu^{\lambda}=\mu_{+}^{\lambda}+\mu_{-}^{\lambda}, \\
\nu^{\lambda}=\nu_{+}^{\lambda}+\nu_{-}^{\lambda}, \\
\theta^{\lambda}=\theta_{+}^{\lambda}+\theta_{-}^{\lambda} .
\end{gathered}
$$

When $\delta v \rightarrow 0, \theta^{\lambda} \rightarrow 0$ and $\mu^{\lambda}, \nu^{\lambda} \rightarrow 1$, so we reproduce the right results for the zero SO case.

For finite SO coupling, $\delta v$ appears as a parameter which plays a role in determining the slowest decaying correlation function and which are the divergent susceptibilities. In Fig. 1 we observe, as an example, the behavior of the exponents

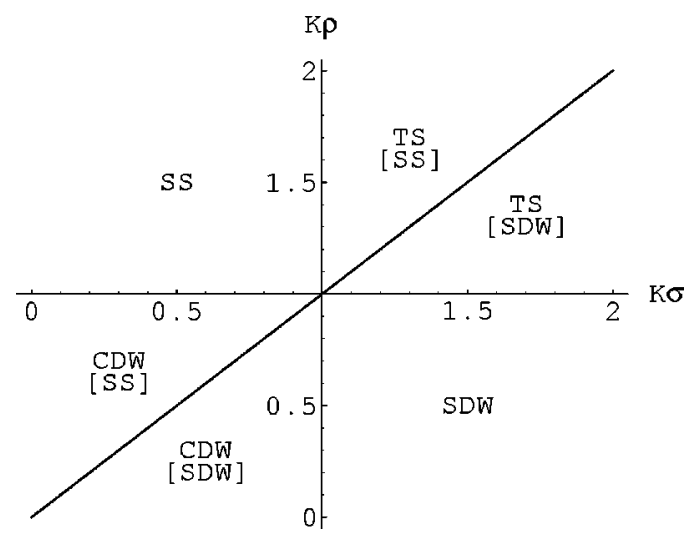

FIG. 2. Phase diagram in $K_{\rho}-K_{\sigma}$ space. The phase in brackets is the subdominant one, which becomes dominant for strong enough SO coupling.
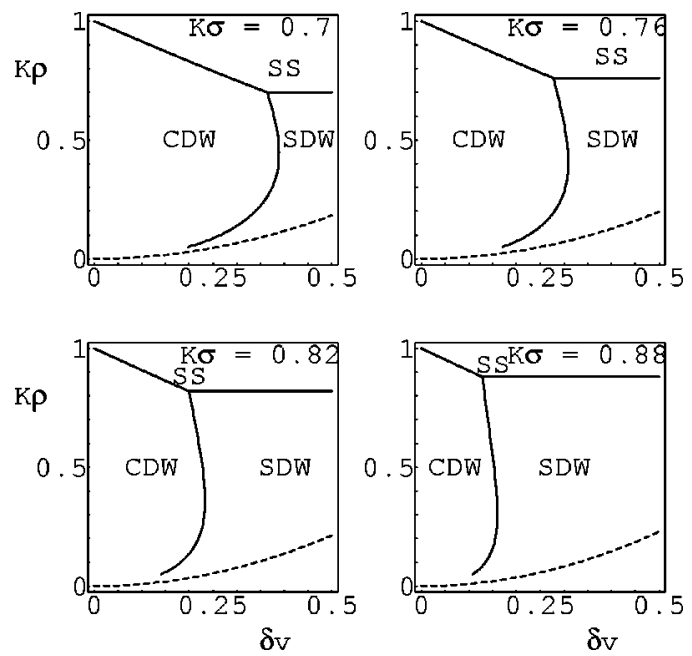

FIG. 3. Phase diagram in $K_{\rho}-\delta v$ space for $v_{\rho}=1.2 v_{0}, v_{\sigma}$ $=0.8 v_{0}$, and different values of $K_{\sigma} . \delta v>\delta v_{\sigma}$ below the dotted line and metamagnetism occurs.

as a function of $\delta v$ for $v_{\rho}=1.2 v_{0}, v_{\sigma}=0.8 v_{0}, K_{\rho}=0.6$, and $K_{\sigma}=0.85$. For $\delta v$ small, CDW fluctuations are dominant, but for $\delta v \gtrsim 0.16 v_{0}$ the SDW, $x y$ correlations decay slower. For small $\delta v$, CDW and SDW fluctuations are the only diverging susceptibilities for $T \rightarrow 0$, but for $\delta v \geqslant 0.25 v_{0}, \alpha_{\mathrm{SS}}$ becomes positive and $\chi_{\mathrm{SS}}$ divergent for $T \rightarrow 0$. Calculations of the electron band structure modified by SO coupling show that these values of $\delta v$ should correspond to typical Q1DES's. ${ }^{12}$

A careful analysis of the exponents allows us to construct a phase diagram in $K_{\rho}-K_{\sigma}$ space (Fig. 2). In each region we indicate the dominant fluctuation for small $\delta v$ and in brackets the dominant one for stronger $\delta v$. Other subdominant fluctuations are not indicated. Cross sections of the phase diagram are shown in Fig. 3. In this plot the $K_{\rho}-\delta v$ space can be observed for $K_{\rho}<1$ and different values of $K_{\sigma}$. For small $\delta v$, CDW fluctuations dominate and for stronger $\delta v$ the system can be either in the SDW or in the SS phase depending on the values of $K_{\rho}$ and $K_{\sigma}$. In the region below the dotted line, $\delta v>\delta v_{\sigma}$, the static spin susceptibility becomes negative and metamagnetism takes place

In conclusion, we have computed correlation functions for a model of one-dimensional correlated electrons with SO coupling. This coupling destroys the spin-charge separation as was shown in Ref. 13 and modifies the exponents of correlation decay. As a consequence the phase diagram gets modified. For strong enough SO coupling, it changes the dominant fluctuation and makes new susceptibilities diverge for $T \rightarrow 0$. How logarithmic corrections originated in irrelevant backscattering and/or umklapp terms modify these results is an interesting problem, the subject of future work.

This work was partially supported by the Consejo Nacional de Investigaciones Científicas y Técnicas (CONICET) and Universidad Nacional de La Plata (UNLP), Argentina. I am grateful to Carlos Naón for useful comments and discussions and for encouraging me to write this article. 
*Electronic address: iucci@fisica.unlp.edu.ar

${ }^{1}$ M. Bockrath, D. Cobden, J. Lu, A. Rinzler, R. Smalley, L. Balents, and P. Mc-Euen, Nature (London) 397, 598 (1999).

${ }^{2}$ A.J. Heeger, .S.A. Kivelson, J.R. Schrieffer, and W.P. Su, Rev. Mod. Phys. 60, 781 (1988).

${ }^{3}$ S. Tarucha, T. Honda, and T. Saku, Solid State Commun. 94, 413 (1995).

${ }^{4}$ S. Tomonaga, Prog. Theor. Phys. 5, 544 (1950); J. Luttinger, J. Math. Phys. 4, 1154 (1963); D. Mattis and E. Lieb, ibid. 6, 304 (1965).

${ }^{5}$ J. Voit, Rep. Prog. Phys. 58, 977 (1995).

${ }^{6}$ J.B. Miller, D.M. Zumbuhl, C.M. Marcus, Y.B. Lyanda-Geller, D. Goldhaber-Gordon, K. Campman, and A.C. Gossard, Phys. Rev. Lett. 90, 076807 (2003).

${ }^{7}$ T. Hassenkam, S. Pedersen, K. Baklanov, A. Kristensen, C.B. Sorensen, P.E. Lindelof, F.G. Pikus, and G.E. Pikus, Phys. Rev. B 55, 9298 (1997).

${ }^{8}$ J. Luo, H. Munekata, F.F. Fang, and P.J. Stiles, Phys. Rev. B 41, 7685 (1990).

${ }^{9}$ J. Nitta, T. Akazaki, H. Takayanagi, and T. Enoki, Phys. Rev. Lett. 78, 1335 (1997).

${ }^{10}$ G. Bergmann, Phys. Rep. 107, 1 (1984).

${ }^{11}$ U. Meirav, M.A. Kastner, M. Heiblum, and S.J. Wind, Phys. Rev. B 40, 5871 (1989).
${ }^{12}$ A.V. Moroz and C.H.W. Barnes, Phys. Rev. B 60, 14272 (1999); 61, R2464 (2000).

${ }^{13}$ A.V. Moroz, K.V. Samokhin, and C.H.W. Barnes, Phys. Rev. Lett. 84, 4164 (2000); Phys. Rev. B 62, 16900 (2000).

${ }^{14}$ J. Solyom, Adv. Phys. 28, 209 (1979).

${ }^{15}$ V. J. Emery, in Highly Conducting One-Dimensional Solids, edited by J. T. Devreese et al. (Plenum, New York, 1979), p. 327.

${ }^{16}$ T. Giamarchi and H.J. Schulz, Phys. Rev. B 39, 4620 (1989); I. Affleck, D. Gepner, T. Ziman, and H. Schulz, J. Phys. A 22, 511 (1989).

${ }^{17}$ J. Voit, Phys. Rev. B 45, 4027 (1992).

${ }^{18}$ F.D.M. Haldane, J. Phys. C 14, 2585 (1981); J. von Delft and H. Schoeller, Ann. Phys. (Leipzig) 7, 225 (1998).

${ }^{19}$ J. Drut and D. Cabra, J. Phys.: Condens. Matter 15, 1445 (2003).

${ }^{20}$ K. Penc and F. Mila, Phys. Rev. B 49, 9670 (1994).

${ }^{21}$ M. Ogata, M.U. Luchini, S. Sorella, and F.F. Assaad, Phys. Rev. Lett. 66, 2388 (1991).

${ }^{22}$ D. Eckert, K. Ruck, M. Wolf, G. Krabbes, and K.-H. Müller, J. Appl. Phys. 83, 7240 (1998).

${ }^{23}$ C. Gerhardt, K.-H. Mütter, and H. Kröger, Phys. Rev. B 57, 11504 (1998).

${ }^{24}$ V.I. Fernández, A. Iucci, and C.M. Naón, Eur. Phys. J. B 30, 53 (2002). 